\title{
Evaluation of Neon Focused Ion Beam Milling for TEM Sample Preparation
}

\author{
T. C. Pekin*, F. I. Allen* and A. M. Minor*
}

* Department of Materials Science and Engineering, University of California, Berkeley, California 94720, USA and the National Center for Electron Microscopy, Molecular Foundry, Lawrence Berkeley National Laboratory, Berkeley, California 94720, USA

Focused ion beam (FIB) milling is a widely-used technique for transmission electron microscopy (TEM) sample preparation [1]. FIB-based preparation of TEM samples offers a number of unique capabilities, including site-specific preparation over a wide size regime of nanometers to many microns and also the ability to thin inhomogeneous samples with high precision [2]. Coupled with micromanipulators and beam-assisted material deposition, modern commercial FIBs can prepare electron-transparent sections for TEM analysis from virtually any material system [3]-[5]. All ion-beam micromachining techniques rely on material removal by ion-induced sputtering. Typically, commercial FIBs use $30 \mathrm{kV} \mathrm{Ga}$ ions extracted from a liquid metal ion source (LMIS), with the beam current being controlled by the aperture size [6].

In this work, an alternative (neon) focused ion beam generated by a gas-field ion source (GFIS) is evaluated for the preparation of electron-transparent specimens. GFIS beams are potentially advantageous for TEM sample preparation when compared to a Ga FIB for two reasons. First, due to the precise and controlled nature of the ion source, as well as more specialized optics, the ion probe size is very small, $\sim 0.5 \mathrm{~nm}$ for He and $\sim 2 \mathrm{~nm}$ for $\mathrm{Ne}[7]$. The microscope's precision is further aided by the profiles of the beams generated, which, while still Gaussian, have much smaller beam tails than the Ga LMIS beam [8]. Secondly, as He and Ne are noble gases with low atomic weights, they do not form alloys in metallic materials or concentrate into precipitates that could obscure imaging in the TEM. Here we have chosen to focus on Ne GFIS beams rather than He, due to the more efficient sputtering by the heavier ion.

For direct comparison, a Ga LMIS and Ne GFIS are used to prepare electron-transparent sections of Si and an Al alloy. Diffraction-contrast TEM imaging and energy dispersive X-ray spectroscopy are used to evaluate the relative damage induced by the two beams, and cross-sections of milled trenches are examined to compare the implantation depth with theoretical predictions from Monte Carlo simulations.

The results show that the amount of damage found in the Ne-milled lamellae was comparable to the damage induced by Ga FIB, which can be seen in Figure 1. Ne bubbling was seen beneath the milling patterns in the samples shown in Figure 2, confirming previous experiments, but was not seen in the thinned lift-outs, which were milled under glancing incidence conditions. The Ne implantation depth matched SRIM calculations very well. In certain circumstances of TEM sample preparation, Ne milling appears to be a valid alternative for when Ga FIB will not suffice. Situations in which this may occur include scenarios when trying to avoid sample contamination by implanted $\mathrm{Ga}$, or cases where the larger Ga probe size does not have the accuracy required for the finest milling patterns. However, our results show that Ne-milling does not avoid beam-induced defect damage in FIB-prepared TEM samples, with similar amounts of damage as induced by Ga-based FIB preparation [9].

[1] L. A. Giannuzzi and F. A. Stevie, Micron 30 (1999) pp. 197-204.

[2] S. Kim, G. Liu, and A. M. Minor, Microscopy Today 17 (2009) pp. 20-23.

[3] L. A. Giannuzzi et al, Microscopy Research and Technique 41 (1998) pp. 285-290. 
[4] S. Kim et al, Ultramicroscopy 111 (2011) pp. 191-9.

[5] R. M. Langford and A. K. Petford-Long, Journal of Vacuum Science and Technology A 19 (2001) p. 2186.

[6] P. R. Munroe, Materials Characterization 60 (2009) pp. 2-13.

[7] S. Tan et al, Journal of Vacuum Science and Technology B 29 (2011) p. $06 \mathrm{~F} 604$.

[8] L. Scipioni et al, Journal of Vacuum Science and Technology B 28 (2010) p. C6P18.

[9] The authors acknowledge support from the National Science Foundation CMMI/MoM program under GOALI Grant 1235610. Portions of this work were performed as a user project at the Molecular Foundry at Lawrence Berkeley National Laboratory, which is supported by the U.S. Department of Energy under Contract \# DE-AC02-05CH11231. The Ne milling was performed using a Zeiss Orion NanoFab microscope in the Biomolecular Nanotechnology Center/QB3 at the University of California, Berkeley.

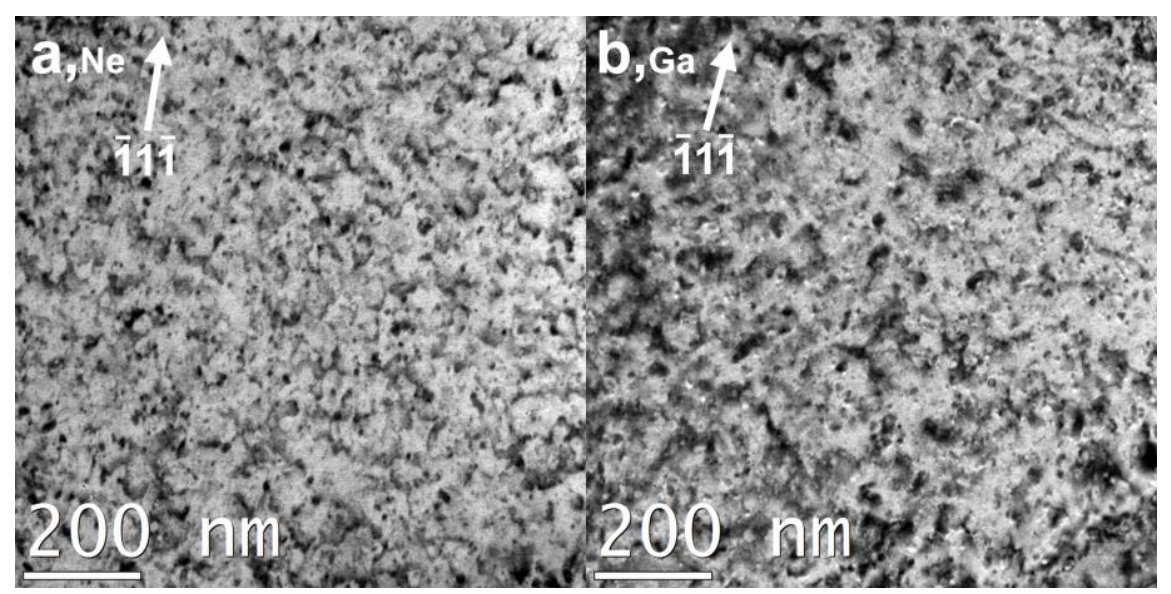

Figure 1. a) Black dot damage and dislocation loops induced by the $10 \mathrm{kV}$ Ne ion beam in Al. b) Similar damage from a $30 \mathrm{kV}$ Ga ion beam in the same alloy. Both TEM images were taken with identical diffraction conditions.
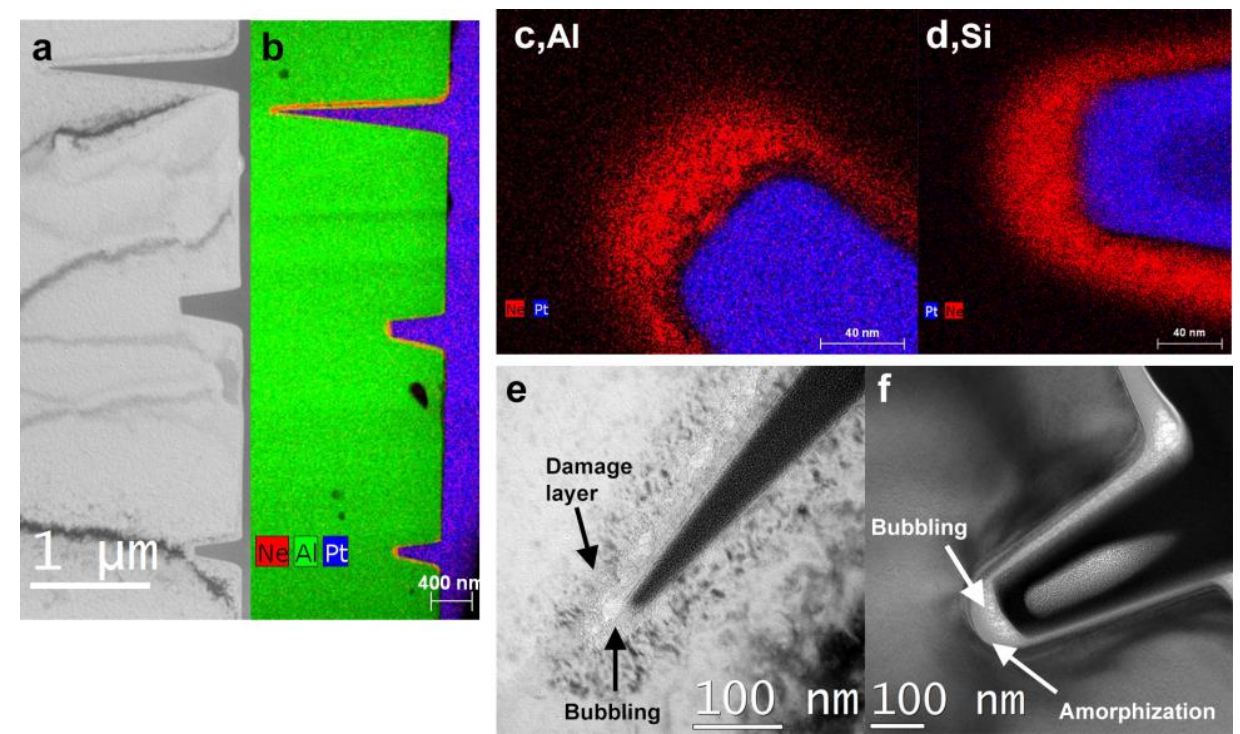

Figure 2. a) Overview bright-field TEM image of milled lines and b) corresponding EDS map for Al sample. Higher resolution EDS maps of Ne implantation in c) Al and d) Si. Bright-field TEM image showing evidence of a Ne damage layer and bubbling in e) Al, and bubbling and amorphization in f) Si. 